\title{
Possible resolution of the domain wall problem in the NMSSM
}

\author{
Anupam Mazumdar, ${ }^{1,2}$ Ken'ichi Saikawa, ${ }^{3,4}$ Masahide Yamaguchi, ${ }^{3}$ and Jun'ichi Yokoyama ${ }^{5-7}$ \\ ${ }^{1}$ Consortium for Fundamental Physics, Lancaster University, Lancaster LA1 4YB, United Kingdom \\ ${ }^{2}$ IPPP, Durham University, Durham DH1 3LE, United Kingdom \\ ${ }^{3}$ Department of Physics, Tokyo Institute of Technology, 2-12-1 Ookayama, Meguro-ku, Tokyo 152-8551, Japan \\ ${ }^{4}$ Deutsches Elektronen-Synchrotron DESY, Notkestrasse 85, 22607 Hamburg, Germany \\ ${ }^{5}$ Research Center for the Early Universe (RESCEU), Graduate School of Science, The University of Tokyo, \\ Tokyo 113-0033, Japan \\ ${ }^{6}$ Department of Physics, Graduate School of Science, The University of Tokyo, Tokyo 113-0033, Japan \\ ${ }^{7}$ Kavli Institute for the Physics and Mathematics of the Universe (Kavli IPMU), UTIAS, WPI, \\ The University of Tokyo, Kashiwa, Chiba 277-8568, Japan
}

(Received 18 November 2015; published 5 January 2016)

\begin{abstract}
We discuss a possibility that the domain wall problem in the next-to-minimal supersymmetric standard model is alleviated without introducing a small explicit $Z_{3}$ breaking term by analyzing the evolution of the singlet scalar field within an inflationary paradigm. The singlet scalar field which explains the $\mu$-term tracks a time-varying minimum of the effective potential after inflation and slowly rolls down to its global minimum if there exist sufficiently large negative Hubble-induced corrections on the effective potential for the singlet field, which arise through supergravity. As a consequence, the whole Universe is confined within a single domain during and after inflation, which prevents the formation of domain walls. This will further constrain the history of the early Universe along with the Higgs-singlet coupling.
\end{abstract}

DOI: 10.1103/PhysRevD.93.025002

\section{INTRODUCTION}

Supersymmetry (SUSY) is the most popular and plausible paradigm to resolve the hierarchy problem in nature between the grand unification scale and the electroweak scale; for a review, see $[1,2]$. The SUSY is also highly attractive from cosmological viewpoints [3]. It provides an appropriate candidate for cold dark matter in terms of the lightest SUSY particle (LSP) [4]. It is also useful to preserve the flatness of the inflaton potential against radiative corrections. Indeed one can find a number of candidates for the inflaton in models with SUSY or supergravity (see, e.g., [5-7] for reviews), making use of a gauge-singlet multiplet [8-10], gauge invariant flat directions [11,12] (see also [13]), or Higgs fields having a nonminimal scalar-curvature coupling $[14,15]$. Finally, an efficient mechanism of baryogenesis has been proposed in SUSY, making use of flat directions [16].

The minimal version of the SUSY standard model (SM), also known as MSSM, contains one dimensionful parameter in the superpotential, namely, the $\mu$-term, i.e., $\mu H_{u} H_{d}$, where the SU(2) doublets $H_{u}$ and $H_{d}$ yield masses to uplike and downlike quarks as they acquire vacuum expectation values (VEVs), respectively. It is desirable that the origin of such a dimensionful parameter as well as its magnitude, $\mu \sim \mathcal{O}(\mathrm{TeV})$, be explained by a more fundamental theory. Along this line, it has been proposed to extend MSSM to incorporate an additional singlet chiral superfield $S$, which dynamically generates the $\mu$-term $[17,18]$. This is also known as next-to-MSSM (NMSSM); for a review, see [19].

Terms dependent on an absolute gauge singlet, $S$, in the renormalizable superpotential for the NMSSM read

$$
W=\lambda S H_{u} H_{d}+\frac{\kappa}{3} S^{3}+W_{\mathrm{MSSM}},
$$

where $W_{\text {MSSM }}$ represents the usual Yukawa interactions between Higgs doublets and quarks/leptons in the MSSM, and $\lambda$ and $\kappa$ are dimensionless couplings. In this model, typically a discrete $Z_{3}$ symmetry is imposed under which all chiral superfields $\Phi$ transform as $\Phi \rightarrow e^{2 \pi i / 3} \Phi$. Such a symmetry guarantees the absence of terms like $\propto S$ and $\propto S^{2}$ as well as the $\mu$-term in the MSSM.

The VEV of $\langle S\rangle$, however, will spontaneously break the $Z_{3}$ symmetry, such that $\mu=\lambda\langle S\rangle \sim \mathcal{O}\left(10^{2}-10^{3}\right) \mathrm{GeV}$ in order to explain the low-scale SUSY spectrum and the observed Higgs mass. This symmetry breaking also poses an intriguing problem and a challenge, which leads to the formation of domain walls in the Universe. Although domain walls with tiny energy scale may yield some interesting cosmological consequences [20] including mild acceleration of cosmic expansion [21], in the present case, their energy scale is so high that if they persist in the late Universe, they simply cause cosmological disasters overdominating the energy density of the Universe [22].

In the literature, in order to evade this problem, it is usually assumed that the $Z_{3}$ symmetry is an accidental symmetry and there exists some explicit symmetry breaking term, which leads to the collapse of domain walls at late times. ${ }^{1}$ The purpose of the present paper is to provide an

\footnotetext{
${ }^{1}$ For instance, the small explicit $Z_{3}$ breaking term can be obtained by imposing a discrete subgroup of $U(1)_{R}$ symmetry [23]. The large explicit $Z_{3}$ breaking term can also be generated in the context of superconformal embedding of NMSSM into supergravity [15].
} 
alternative explanation to avoid the domain wall problem in the context of inflationary cosmology rather than an explicit symmetry breaking term employed so far in the literature. We will see that the formation of domain walls can be alleviated if there exist sufficiently large negative supergravity corrections proportional to the Hubble parameter in the effective potential for the singlet scalar field during and after inflation. ${ }^{2}$ Since the singlet field tracks a time-varying minimum of the effective potential after inflation, we call this scenario the tracking mechanism.

The outline of the paper is as follows: In Sec. II, we discuss the domain wall formation in the inflationary context. Then possible effects caused by supergravity corrections are described in Sec. III. In Sec. IV, we analyze the evolution of the singlet field with a negative Hubbleinduced mass and derive the conditions for the tracking mechanism to work. Initial conditions for the singlet field during inflation are also discussed in Sec. V. In Sec. VI, we take account of finite temperature corrections for the evolution of the singlet field after inflation and obtain some conditions on the Higgs-singlet coupling and the reheating temperature in order to avoid the domain wall formation. The consequences for cosmology in the NMSSM are briefly discussed in Sec. VII. Finally, Sec. VIII is devoted to the conclusion and discussions.

\section{NMSSM DOMAIN WALLS AND INFLATION}

In order to address this issue, let us first note that from $\mu=\lambda\langle S\rangle \sim \mathcal{O}\left(10^{2}-10^{3}\right) \mathrm{GeV}$, we see that $\langle S\rangle$ can be much larger than the weak scale if the coupling $\lambda$ is sufficiently small. In this case, the scalar potential can be written as

$$
V \simeq \kappa^{2}|S|^{4}+m_{S}^{2}|S|^{2}+\left(\frac{\kappa}{3} A_{\kappa} S^{3}+\text { H.c. }\right)
$$

where $m_{S}^{2}$ and $A_{\kappa}$ are the soft SUSY breaking mass parameters. ${ }^{3}$ From the above form of the potential, we can estimate the VEV of $S$ at the global minimum as

$$
\langle S\rangle_{\text {global }} \simeq-\frac{A_{\kappa}}{4 \kappa}\left(1+\sqrt{1-\frac{8 m_{S}^{2}}{A_{\kappa}^{2}}}\right) .
$$

\footnotetext{
${ }^{2}$ A similar scenario was considered in Ref. [24], in which a new gauge singlet field is introduced in addition to the NMSSM field content and this new scalar field acquires a large Hubbleinduced mass. Instead of introducing such an extra symmetry breaking field, in this paper we discuss a possibility that the domain wall problem is avoided solely due to the dynamics of the singlet scalar field $S$ in NMSSM.

${ }^{3}$ In principle, superpotential terms such as $W \subset \mathcal{O}(1) S^{n} / M_{\mathrm{Pl}}^{n-3}$ would also appear, where $n=6,9, \cdots$, and $M_{\mathrm{Pl}} \simeq 2.4 \times$ $10^{18} \mathrm{GeV}$ is the reduced Planck mass. In this paper we are ignoring these higher order contributions to the superpotential and the potential.
}

Note that the magnitude of $A_{\kappa}$ must be slightly larger than that of $m_{S}$ in order to guarantee the existence of the global minimum with $\langle S\rangle \neq 0[25]$ :

$$
A_{\kappa}^{2} / m_{S}^{2} \gtrsim \mathcal{O}(10) .
$$

Furthermore, $\lambda$ and $\kappa$ cannot have a large hierarchy if the magnitudes of all dimensionful parameters $\mu, m_{S}$, and $A_{\kappa}$ are close to the weak scale. In this paper, we assume $\lambda \simeq \kappa$ for simplicity.

Before discussing the resolution of the problem, let us describe how likely the formation of domain walls occurs in the inflationary context. For simplicity, we assume that inflation is driven by a potential energy of some scalar field, called inflaton, and that the inflaton sector does not embody the $S$ superfield, or as a matter of fact other MSSM superfields. A naive expectation is that the domain wall formation is avoidable if the thermal corrections to the effective potential of the singlet scalar remain irrelevant after inflation such that the $Z_{3}$ symmetry is never restored, but this simple observation turns out to be insufficient when we carefully consider the evolution of the singlet scalar during and after inflation due to the reasons discussed below.

During inflation, quantum mechanically induced vacuum fluctuations significantly displace any light scalar field whose effective mass is smaller than the Hubble parameter $H_{\text {inf }}$ because it obtains quantum fluctuations of order $H_{\text {inf }} / 2 \pi$ within each Hubble time [26-28]. After a sufficiently large number of $e$-folds of inflation, a free real scalar field $\phi$ with mass $m_{\phi}$ acquires long-wave fluctuations with dispersion

$$
\left\langle\phi^{2}\right\rangle=\frac{3 H_{\mathrm{inf}}^{4}}{8 \pi^{2} m_{\phi}^{2}} .
$$

The dispersion of a scalar field with a more complicated potential can be calculated by the stochastic inflation method [29] but the typical field amplitude can be estimated by simply requiring that the typical value of its potential energy density takes a value $\mathcal{O}\left(H_{\text {inf }}^{4}\right)$.

This fact implies that the VEV of the $S$ field during inflation is generically different from the value $\langle S\rangle_{\text {global }}$, Eq. (2), at the global minimum of the low-energy effective potential because the bare mass of $S$ field is $m_{S} \sim \mathcal{O}(\mathrm{TeV}) \ll H_{\text {inf }}$. After inflation, the $S$ field starts to oscillate, reducing its amplitude with time. It eventually falls into one of the global minima, whose phase is related by the $Z_{3}$ transformation to that of other minima.

Note, however, that the final value of the phase of $\langle S\rangle$ can differ at each spatial point because of the existence of the field fluctuations $\delta S(x)$. These field variations originate from quantum fluctuations during inflation, and they may be enhanced once the $S$ field starts to oscillate due to the parametric resonance effect; see [30]. The enhancement occurs both in the radial and angular direction of the 
complex scalar $S$, since the $A_{\kappa}$ term mixes them. As a result, different domains are created within the horizon scale, and domain walls are formed around their boundary. The above discussion suggests that the formation of domain walls is almost inevitable unless the $S$ field is stabilized at the minimum of the effective potential during inflation.

\section{SUPERGRAVITY CORRECTION AND THE EVOLUTION OF $S$}

In this paper, we shall argue that the resolution of this problem is also achieved within the inflationary paradigm (For a recent review of inflation, see, e.g., [31].) The key ingredient is an effective mass of the form $-c H^{2}$ for the $S$ field, where $H$ is the Hubble parameter at a given time. Such a mass term generically arises via a Plancksuppressed interaction in the framework of supergravity [32]; see also [3,5-7]. Since the value of the coefficient $c$ depends on the details of the Kähler terms [33], it is fair to treat it as a free parameter to keep the discussion model independent. According to its value, we may consider the following three possibilities:

(i) If there exists no Hubble-induced mass $(c=0)$ : In this case, $S$ is expected to take a large value $S \sim$ $H_{\text {inf }} / \sqrt{\kappa}$ during inflation due to the accumulation of long wave quantum fluctuations. It starts oscillation as the Hubble parameter gets smaller than the effective mass of $S$ after inflation. Then even in case a motion along the angular direction was suppressed initially, as it crossed the origin, it would start the angular motion and the phase of the scalar field would have a scattered distribution due to the $Z_{3}$ symmetry of the potential. As a result, formation of domain walls is inevitable.

(ii) If the Hubble-induced mass is positive $(c<0)$ : In this case, during inflation the $S$ field is stabilized at the origin. After inflation, it rolls down to the global minimum from the origin when the temperature of the Universe becomes sufficiently low (i.e., the phase transition occurs), and again domain walls are created through the Kibble mechanism.

(iii) If the Hubble-induced mass is negative $(c>0)$ : In this case, during inflation the $S$ field takes a value larger than $\langle S\rangle_{\text {global }}$, very similar to our Fig. 1. As we will see below, there is a possibility to avoid the domain wall formation in this case. Indeed if $S$ sits on its potential minimum at each time and adiabatically traces its time evolution until the field relaxes to $\langle S\rangle_{\text {global }}$, we may avoid the domain wall formation.

From here onwards, we will only consider the last case with a negative Hubble-induced mass $(c>0)$ to see if the above-mentioned tracking scenario works. We can write the effective potential for the $S$ field during inflation as

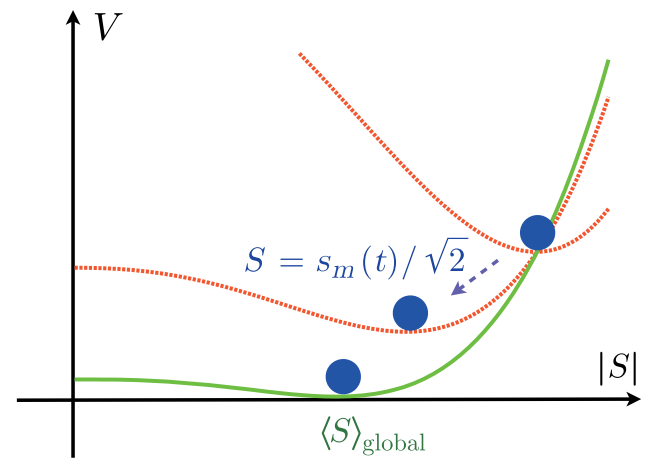

FIG. 1. Sketch of the tracking mechanism. The red dotted lines represent the form of the effective potential with the negative mass term $-\tilde{c} H^{2}|S|^{2}$ at early times, and the green solid line represents that at the present time where the Hubble-induced term becomes irrelevant. The location of the minimum varies with time, which is indicated by blue circles.

$$
V=-c H_{\mathrm{inf}}^{2}|S|^{2}-\left(\frac{\kappa}{3} c^{\prime} H_{\mathrm{inf}} S^{3}+\text { H.c. }\right)+\kappa^{2}|S|^{4},
$$

where terms dependent on $H_{\text {inf }}$ are induced by the inflaton's potential through the supergravity effect. Here we have ignored the soft SUSY breaking contributions, since $m_{S}, A_{\kappa} \ll H_{\text {inf }}$, and the possible interaction terms with two Higgses, since they are stabilized at the origin due to the large field value of $S$. The cubic term of the form $\kappa c^{\prime} H S^{3} / 3$ with $c^{\prime}$ being another coefficient plays an important role in stabilizing the phase of $S$ to the minimum of the potential.

Terms dependent on the Hubble parameter $H(t)$ induced by supergravity effects are present even in the field oscillation regime after inflation:

$$
V(S)=-\tilde{c} H^{2}|S|^{2}-\left(\frac{\kappa}{3} \tilde{c^{\prime}} H S^{3}+\text { H.c. }\right)+\kappa^{2}|S|^{4} .
$$

Here the coefficients, $\tilde{c}$ and $\tilde{c^{\prime}}$, may take somewhat smaller values than $c$ and $c^{\prime}$ during inflation, respectively, because in this regime the kinetic energy is comparable to the potential energy. Hereafter we assume that all these four parameters are real and positive, and that $\mathcal{O}(c) \simeq \mathcal{O}(\tilde{c})$ and $\mathcal{O}\left(c^{\prime}\right) \simeq \mathcal{O}\left(\tilde{c^{\prime}}\right)$ for simplicity. It should be noticed that (the origins of) the phases of the $A$ terms in Eqs. (5) and (6) might not be identical if the Kähler structures responsible for the $A$ terms are changed. Since the Kähler structures remain unchanged during inflation and inflaton oscillation due to the energy dominance of the inflaton field, both phases are expected to coincide. On the other hand, they may change generally after the inflaton decays and radiation dominates the energy density of the Universe. Here we assume both phases coincide for simplicity. 


\section{TRACKING OF THE INSTANTANEOUS MINIMUM FOR THE $S$ FIELD}

In order for the tracking scenario to work, we must address the following two issues, apart from thermal effects which will be discussed later. First, it must trace the time evolution of the minimum adiabatically in the postinflationary Universe when the effective potential is given by Eq. (6). Second, the initial fluctuations of the singlet field generated during inflation are small enough not to create different domains at a later time. In this section we discuss the former issue.

In terms of $S \equiv s e^{i \theta} / \sqrt{2}(s \geq 0)$, Eq. (6) is expressed as

$$
V(s, \theta)=-\frac{\tilde{c}}{2} H^{2} s^{2}-\frac{\kappa \tilde{c}^{\prime}}{3 \sqrt{2}} H s^{3} \cos 3 \theta+\frac{\kappa^{2}}{4} s^{4} .
$$

The angular direction has one of the minima at $\theta=0$, where the radial direction feels the potential

$$
V(s, 0)=-\frac{\tilde{c}}{2} H^{2} s^{2}-\frac{\kappa \tilde{c^{\prime}}}{3 \sqrt{2}} H s^{3}+\frac{\kappa^{2}}{4} s^{4},
$$

which is minimized at

$$
s=\frac{1}{2 \sqrt{2} \kappa}\left(\tilde{c^{\prime}}+\sqrt{{\tilde{c^{\prime}}}^{2}+8 \tilde{c}}\right) H(t) \equiv s_{m}(t) .
$$

Neglecting the motion in the phase direction and fixing as $\theta=0$, we obtain the equation for the radial direction:

$$
\ddot{s}+3 H \dot{s}-\tilde{c} H^{2} s-\frac{1}{\sqrt{2}} \tilde{\kappa c^{\prime}} H s^{2}+\kappa^{2} s^{3}=0 .
$$

By introducing a new variable $\xi=s / s_{m}$, the above equation can be rewritten as

$$
\begin{aligned}
& \xi^{\prime \prime}+F \xi^{\prime}+(H t)^{2} \xi \\
& \quad \times\left[\left(\tilde{c}+\frac{\xi}{8}\left(\tilde{c^{\prime}}+\sqrt{\tilde{c}^{\prime 2}+8 \tilde{c}}\right)^{2}\right)(\xi-1)+G\right]=0,
\end{aligned}
$$

where

$$
\begin{aligned}
F & \equiv(H t)\left(2 \frac{\dot{H}}{H^{2}}+3\right)-1, \\
G & \equiv \frac{\ddot{H}}{H^{3}}+3 \frac{\dot{H}}{H^{2}},
\end{aligned}
$$

and $\xi^{\prime} \equiv d \xi / d \ln t=t \dot{\xi}$. The tracking scenario works only if $\xi \simeq 1$ holds throughout its evolution.

Note that for $a(t) \propto t^{p}$, where $a(t)$ represents the scale factor of the Universe at a given time, we have $F=$ $3(p-1)$ and $G=(2-3 p) / p^{2}$. Just after inflation the inflaton field oscillates around the minimum of its potential behaving like a matter component, which leads to $p=2 / 3$ for the cosmic expansion. Then we have $G=0$, and $\xi=1$ becomes a solution of the field equation [Eq. (11)]. However, this solution is unstable since the damping is negative, $F=-1<0$. In other words, if there exists a deviation from $\xi=1$ initially, it grows with time and spoils the tracking mechanism when the deviation becomes $\mathcal{O}(1)$.

The instability described above can be alleviated if the coefficient $\tilde{c}$ or $\tilde{c^{\prime}}$ is sufficiently large. Note that just before the end of inflation $\left(|\dot{H}| / H^{2} \lesssim 1\right)$ the damping is positive $(F>0)$, and the field variable $\xi$ exponentially converges into the value determined by setting the bracket in the lefthand side of Eq. (11) to zero, which reads

$$
\xi \simeq 1-\frac{4 G}{\left(\tilde{c^{\prime}}+\sqrt{\tilde{c}^{\prime 2}+8 \tilde{c}}\right) \sqrt{{\tilde{c^{\prime}}}^{2}+8 \tilde{c}}}
$$

for $\tilde{c}, \tilde{c^{\prime}} \gg 1$. The effects of quantum fluctuations will be discussed and shown to be negligible in the next section. Since $G \sim \mathcal{O}(1)$ just before the end of inflation, this fact implies that at this epoch the value of $\xi$ deviates from $\xi=1$ by the following quantity:

$$
\delta \xi_{i} \sim \mathcal{O}\left(\tilde{c}^{-1}, \tilde{c}^{\prime-2}\right) .
$$

After inflation, the initial deviation $\delta \xi_{i}$ starts to grow. To see how it grows, let us substitute $\xi=1+\delta \xi$ with $\delta \xi \ll 1$ into Eq. (11). Assuming that $F=-1, G=0$, and $H t=2 / 3$ during the inflaton-oscillation dominated phase, we have

$$
\delta \xi^{\prime \prime}-\delta \xi^{\prime}+\frac{1}{9}\left(\tilde{c^{\prime}}+\sqrt{\tilde{c}^{\prime 2}+8 \tilde{c}}\right) \sqrt{\tilde{c^{\prime 2}}+8 \tilde{c}} \delta \xi=0
$$

where we neglected the terms of higher order in $\delta \xi$. The above equation implies that the deviation grows with time as $\delta \xi \propto t^{1 / 2}$ for $\tilde{c}, \tilde{c}^{\prime} \gg 1$.

In order to guarantee that the deviation from the minimum $s=s_{m}$ remains small throughout its evolution, we require the following condition:

$$
\left|\delta \xi\left(t_{g}\right)\right|=\left(\frac{t_{g}}{t_{i}}\right)^{\frac{1}{2}}\left|\delta \xi_{i}\right|=\left(\frac{s_{m, \text { inf }}}{s_{m, \text { global }}}\right)^{\frac{1}{2}}\left|\delta \xi_{i}\right| \ll 1,
$$

where $t_{g}$ and $t_{i}$ represent the time at which the $S$ field reaches the global minimum and that at the end of inflation, respectively. In the second equality of the above equation, we used the fact that $s_{m}(t) \propto H(t) \propto 1 / t$. Here $s_{m, \text { inf }}$ corresponds to the value at the end of inflation,

$$
s_{m, \text { inf }} \equiv \frac{1}{2 \sqrt{2} \kappa}\left(c^{\prime}+\sqrt{c^{\prime 2}+8 c}\right) H_{\mathrm{inf}}
$$

and $s_{m, \text { global }} \equiv \sqrt{2}\langle S\rangle_{\text {global }}$ corresponds to that at the global minimum [see Eq. (2)]. The condition given by Eq. (16) implies that 
TABLE I. Lower bounds on the coefficients of the Hubbleinduced corrections given by Eq. (18) for some choices of the energy scale of inflation $V_{\text {inf }}^{1 / 4}$. Here we use the relation $H_{\text {inf }}^{2}=$ $V_{\text {inf }} / 3 M_{\mathrm{Pl}}^{2}$ and the value for the soft parameter $\left|A_{\kappa}\right|=1 \mathrm{TeV}$.

\begin{tabular}{lcc}
\hline \hline$V_{\text {inf }}^{1 / 4}$ & $2 \times 10^{12} \mathrm{GeV}$ & $2 \times 10^{15} \mathrm{GeV}$ \\
$H_{\text {inf }}$ & $10^{6} \mathrm{GeV}$ & $10^{12} \mathrm{GeV}$ \\
\hline$c, \tilde{c}$ & $\gtrsim 100$ & $\gtrsim 10^{6}$ \\
$c^{\prime}, \tilde{c^{\prime}}$ & $\gtrsim 10$ & $\gtrsim 10^{3}$ \\
\hline \hline
\end{tabular}

$$
c, \tilde{c} \gg \mathcal{O}\left(\frac{H_{\mathrm{inf}}}{\left|A_{\kappa}\right|}\right)^{\frac{2}{3}} \text { or } \quad c^{\prime}, \tilde{c^{\prime}} \gg \mathcal{O}\left(\frac{H_{\mathrm{inf}}}{\left|A_{\kappa}\right|}\right)^{\frac{1}{3}} .
$$

We summarize some representative values for the coefficients of the Hubble-induced correction terms in Table I. According to the amplitude of the Hubble parameter during inflation (and hence the energy scale of inflation), relatively large coefficients are required. These required values might be much larger than those derived in Ref. [34] in the context of the cosmological moduli problem. This is because there exist both positive and negative Hubble-induced terms in the model considered in [34], in contrast to the scenario in this paper where there exists the negative Hubble-induced term only. In the latter case the dragging of the scalar field is not so efficient as we expect in the former case. Likewise, we expect that the dragging mechanism of Ref. [34] does not relax the moduli problem if the effective potential possesses the negative Hubble-induced term only.

We also note that at the time $t \lesssim t_{g}$, the $S$ field does not rotate in the phase direction as long as the $A_{\kappa}$ term satisfies the condition given by Eq. (3). Then the transition from one vacuum to others related by the $Z_{3}$ transformations is prohibited even after the negative Hubble-induced terms become irrelevant.

\section{INITIAL CONDITION FOR $S$ FIELD DURING INFLATION}

Let us next study the field configuration during inflation, keeping the above parameter values in mind. The potential minimum is located at $s_{m, \text { inf }} \gg H_{\text {inf }} / \kappa$ [see Eq. (17)], and quantum fluctuations around it are estimated as

$$
\left\langle\left(s-s_{m, \text { inf }}\right)^{2}\right\rangle \simeq \frac{3 H_{\mathrm{inf}}^{4}}{8 \pi^{2} m_{s}^{2}}<\frac{3}{16 \pi^{2} c} H_{\mathrm{inf}}^{2} \ll 10^{-3} H_{\mathrm{inf}}^{2},
$$

where

$$
\begin{aligned}
m_{s}^{2} & \equiv \frac{\partial^{2} V}{\partial s^{2}}\left(s_{m, \mathrm{inf}}, 0\right) \\
& =2 c H_{\mathrm{inf}}^{2}+\frac{1}{4}\left(c^{\prime 2}+c^{\prime} \sqrt{c^{\prime 2}+8 c}\right) H_{\mathrm{inf}}^{2}
\end{aligned}
$$

and it is assumed that only the mass term is important. Thus, fluctuations along the radial direction are much smaller than the expectation value $s_{m \text {,inf }}$, and we may study fluctuations along the angular direction by setting $s=s_{m, \text { inf }}$.

The potential for the angular scalar field defined by $\chi \equiv$ $s_{m, \text { inf }} \theta$ is given by

$$
\begin{aligned}
V(\chi) & =-\frac{\kappa c^{\prime}}{3 \sqrt{2}} H_{\mathrm{inf}} s_{m, \mathrm{inf}}^{3} \cos \left(3 \frac{\chi}{s_{m, \mathrm{inf}}}\right) \\
& =\frac{3 \kappa c^{\prime}}{2 \sqrt{2}} H_{\mathrm{inf}} s_{m, \mathrm{inf}} \chi^{2}+\cdots,
\end{aligned}
$$

where the latter expression applies for small $\theta$, and we can read off the mass of $\chi$ as

$$
m_{\chi}^{2}=\frac{3 c^{\prime}}{4}\left(c^{\prime}+\sqrt{c^{\prime 2}+8 c}\right) H_{\mathrm{inf}}^{2} .
$$

Let us estimate the number of domains where fluctuation along the angular direction exceeds $|\theta|=\pi / 3$ to make a domain wall somewhere within our observable Universe using the approximated potential [Eq. (21)] based on the peak theory of random Gaussian fields (Ref. [35]). The desired quantity can be calculated from the correlation function

$$
C\left(\left|z-z^{\prime}\right|\right) \equiv\left\langle\chi(\boldsymbol{z}) \chi\left(\boldsymbol{z}^{\prime}\right)\right\rangle=\frac{3 H_{\mathrm{inf}}^{4}}{8 \pi^{2} m_{\chi}^{2}}\left(H_{\mathrm{inf}}\left|z-\boldsymbol{z}^{\prime}\right|\right)^{-\frac{2 m_{\chi}^{2}}{3 H_{\mathrm{inf}}^{2}}}
$$

and its derivatives at zero lag, such as

$$
\sigma_{0}^{2} \equiv\left\langle\chi^{2}(0)\right\rangle=C(0)=\frac{3 H_{\mathrm{inf}}^{4}}{8 \pi^{2} m_{\chi}^{2}}
$$

$$
\begin{aligned}
\sigma_{1}^{2} & \equiv 3\left\langle\frac{\partial \chi}{\partial z_{x}}(\boldsymbol{z}) \frac{\partial \chi}{\partial z_{x}^{\prime}}\left(z^{\prime}\right)\right\rangle=-\left.3 \frac{C^{\prime}(r)}{r}\right|_{r \rightarrow H_{\mathrm{inf}}^{-1}}=\frac{3 H_{\mathrm{inf}}^{4}}{4 \pi^{2}}, \\
\sigma_{2}^{2} & \equiv 15\left\langle\frac{\partial^{2} \chi}{\partial z_{x}^{2}}(\boldsymbol{z}) \frac{\partial^{2} \chi}{\partial z_{y}^{\prime 2}}\left(z^{\prime}\right)\right\rangle=\left.15\left(\frac{C^{\prime \prime}(r)}{r^{2}}-\frac{C^{\prime}(r)}{r^{3}}\right)\right|_{r \rightarrow H_{\mathrm{inf}}^{-1}} \\
& =\frac{5 H_{\mathrm{inf}}^{4}}{2 \pi^{2}}\left(m_{\chi}^{2}+3 H_{\mathrm{inf}}^{2}\right) .
\end{aligned}
$$

Note that Eq. (23) is applicable for $r \gtrsim H_{\text {inf }}^{-1}$, but it also reproduces the variance with zero lag, taking $r=H_{\mathrm{inf}}^{-1}$. Hence, we take $r \rightarrow H_{\text {inf }}^{-1}$ when we consider the zero-lag limit in stochastic inflation (Ref. [29]).

Then according to Ref. [35], the number density of the $\nu \sigma$ peak is calculated as 


$$
n_{p}(\nu) d \nu=\frac{e^{-\frac{\nu^{2}}{2}}}{(2 \pi)^{2} R_{*}^{3}} G(\gamma, \gamma \nu) d \nu \equiv e^{-f(\nu)} d \nu,
$$

where $G(\gamma, w)$ is a function whose approximate form can be found in Eq. (4.4) of Ref. [35]. Here $\gamma$ and $R_{*}$ are, respectively, given by

$$
\gamma=\left(\frac{6 m_{\chi}^{2}}{10 m_{\chi}^{2}+30 H_{\mathrm{inf}}^{2}}\right)^{\frac{1}{2}}, \quad R_{*}=\frac{3}{\left(10 m_{\chi}^{2}+30 H_{\mathrm{inf}}^{2}\right)^{1 / 2}} .
$$

Assuming that the number of $e$-folds of inflation required to solve the horizon problem is equal to 60 , the number of domains with $|\theta|>\pi / 3$ in the observable Universe today is given by [36]

$$
\begin{aligned}
2 N\left(>\nu_{d}\right) & =2 e^{180} H_{\mathrm{inf}}^{-3} \int_{\nu_{d}}^{\infty} n_{p}(\nu) d \nu \simeq \frac{2 e^{180-f\left(\nu_{d}\right)}}{H_{\mathrm{inf}}^{3} f^{\prime}\left(\nu_{d}\right)}, \\
& \simeq \frac{e^{180-\nu_{d}^{2} / 2}}{2 \pi^{2} \nu_{d}}\left(\frac{10 m_{\chi}^{2}+30 H_{\mathrm{inf}}^{2}}{9 H_{\mathrm{inf}}^{2}}\right)^{\frac{3}{2}} G\left(\gamma, \gamma \nu_{d}\right),
\end{aligned}
$$

where $\nu_{d} \equiv \pi s_{m \text {,inf }} /(3 \sqrt{C(0)})$. Requiring that the above expression should be smaller than unity, we find only a mild constraint on $\kappa$. This can be easily seen from the exponent $-\nu_{d}^{2} / 2=-\pi^{4} c^{\prime}\left(c^{\prime}+\left(c^{\prime 2}+8 c\right)^{1 / 2}\right)^{3} / 72 \kappa^{2}$, which gives a huge negative contribution for $\kappa \ll c^{\prime 2}$.

Therefore to summarize, as long as the condition for the tracking behavior is satisfied, quantum fluctuations during inflation are suppressed both along radial and angular directions, so that the domain wall formation is always avoided.

\section{THERMAL EFFECTS ON $S$ AFTER INFLATION AND CONSTRAINTS}

Finally we consider possible thermal effects on the $S$ field after inflation. As the amplitude $|S|$ decreases with time, interactions with light fields in the thermal bath gradually come into play, which can destroy the $S$ field condensate to produce domain walls. In particular, we must ensure the following two conditions:

(1) Thermal corrections of the form $\sim \lambda^{2} T^{2}|S|^{2}$ should be small enough to satisfy $\lambda^{2} T^{2} \ll \tilde{c} H^{2}$ in order not to affect the tracking evolution of the $S$ field.

(2) The amplitude of thermal fluctuation $\delta S(x) \sim T$ of the $S$ field should be well below that of the background field $s_{m}$; otherwise they may cause transitions into different domains to create domain walls.

Since $s_{m} \sim \sqrt{\tilde{c}} H / \kappa$ and $\lambda \simeq \kappa$, we see that these two requirements lead to the identical condition on the model parameters, which is solely determined by the dynamics of later times, since $H$ decreases faster than $T$ after inflation. Hence, we have only to consider the constraint at the time when $S$ has almost reached the global minimum, i.e., $\tilde{c} H^{2} \sim m_{S}^{2}$. Therefore, we may impose the following condition to avoid destruction of the condensate ${ }^{4}$ :

$$
\frac{\lambda T}{\sqrt{\tilde{c}} H}<0.1 \quad \text { at } \tilde{c} H^{2}=m_{S}^{2} .
$$

Let us quantify Eq. (30) more explicitly by considering the evolution of the Universe after inflation. Just after inflation, the inflaton starts to oscillate around the minimum of its own potential, and it eventually decays into the (MS)SM degrees of freedom to reheat the Universe. There are two distinct possibilities: (1) the $S$ field reaches its global minimum during the inflaton-oscillation dominated phase before reheating is completed, and (2) the $S$ field reaches its global minimum in the radiation dominated epoch after reheating.

In the first scenario, the temperature of the thermal bath is given by $T \simeq\left(H T_{R}^{2} M_{\mathrm{Pl}}\right)^{1 / 4}$ [37], where $T_{R}$ is the reheating temperature. At the relevant epoch we find $T \simeq \tilde{c}^{-1 / 8}\left(m_{S} T_{R}^{2} M_{\mathrm{Pl}}\right)^{1 / 4}$, and Eq. (30) leads to the following bound:

$$
T_{R}<2 \times 10^{3} \operatorname{GeV}^{\frac{1}{4}}\left(\frac{\lambda}{10^{-5}}\right)^{-2}\left(\frac{m_{S}}{1 \mathrm{TeV}}\right)^{\frac{3}{2}} .
$$

Since $T \simeq \tilde{c}^{-1 / 8}\left(m_{S} T_{R}^{2} M_{\mathrm{Pl}}\right)^{1 / 4}>T_{R}$ by assumption, we obtain an upper bound on $T_{R}$ :

$$
T_{R}<5 \times 10^{10} \mathrm{GeV} \tilde{c}^{-\frac{1}{4}}\left(\frac{m_{S}}{1 \mathrm{TeV}}\right)^{\frac{1}{2}} .
$$

If the condition given by Eq. (32) is not satisfied, the $S$ field reaches its global minimum in the radiation dominated epoch after reheating. From the relation $H \sim T^{2} / M_{\mathrm{Pl}}$, we have $T \simeq \tilde{c}^{-1 / 4}\left(m_{S} M_{\mathrm{Pl}}\right)^{1 / 2}$ at $\tilde{c} H^{2}=m_{S}^{2}$, and by using Eq. (30), we obtain

$$
\lambda<2 \times 10^{-9} \tilde{c}^{\frac{1}{4}}\left(\frac{m_{S}}{1 \mathrm{TeV}}\right)^{\frac{1}{2}} .
$$

Note that the above condition makes sense only if there exists a large negative Hubble-induced mass term $-\tilde{c} H^{2}|S|^{2}$ and $A$ terms $-\left(\tilde{\kappa c^{\prime}} H S^{3}+\right.$ H.c. $)$ even after the reheating is completed. The existence of the negative Hubble-induced mass term is foreseeable due to the SUSY breaking

\footnotetext{
${ }^{4}$ To confirm this observation, we have numerically solved the field equation $\ddot{S}+3 H \dot{S}+\partial V / \partial S=0$ with the potential given by $\quad V \simeq-\tilde{c} H^{2}|S|^{2}+\kappa^{2}|S|^{4}+m_{S}^{2}|S|^{2}+\left(\kappa A_{\kappa} S^{3} / 3-\kappa \tilde{c}^{\prime} H S^{3} / 3\right.$ + H.c.), which gives a good approximation around $\tilde{c} H^{2} \gtrsim m_{S}^{2}$. We have confirmed that for reasonable values of the model parameters the $S$ field does not move to other minima if the deviation from $s_{m} / \sqrt{2}$ is less than $\mathcal{O}(10) \%$, which we use as the criterion shown in Eq. (30).
} 


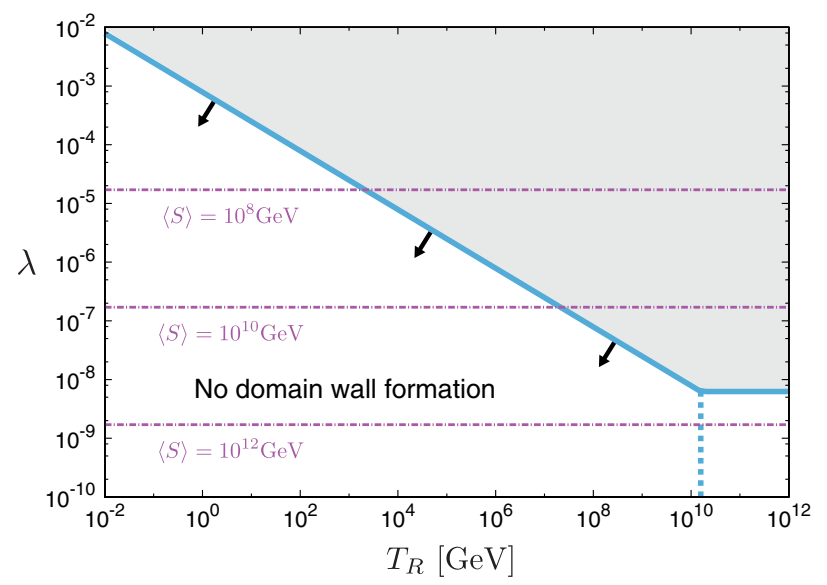

FIG. 2. Conditions to avoid the domain wall formation on the parameter space of $\left(T_{R}, \lambda\right)$. Domain walls are not produced in the region below the blue line corresponding to Eqs. (31) and (33). The region to the right side of the blue dotted line corresponding to Eq. (32) also leads to the domain wall formation if the large negative Hubble-induced mass is absent in the radiation dominated epoch after inflation. We also plot the values of the $\operatorname{VEV}\langle S\rangle$ at the global minimum [Eq. (2)] as purple chain lines. Here we fix the values of other parameters as $\tilde{c}=100, m_{S}=1 \mathrm{TeV}$ and $\left|A_{\kappa}\right|=4 \mathrm{TeV}$.

contributions from the thermal bath [38,39]. The phase of the coefficient $\tilde{c^{\prime}}$ of the Hubble-induced $A$ term can change after reheating since the Kähler structures might change at that epoch, which induces a rotation of the $S$ field in the angular direction. This fact does not spoil the tracking mechanism as long as the magnitudes of the coefficients remain sufficiently large. The reason may be understood as follows: the angular motion induced by the change of the Kähler structures proceeds adiabatically with a Hubble time scale, since the transition to the radiation dominated phase happens smoothly at that epoch. For such a smooth transition, the deviation from the minimum of the effective potential induced by the angular motion is at most $\mathcal{O}\left(\tilde{c}^{-1}, \tilde{c}^{\prime-2}\right)$, which remains negligible for large coefficients.

Figure 2 summarizes our analyses. Combining two cases described above, we show the allowed parameter range for $T_{R}$ and $\lambda(\simeq \kappa)$. From the plot we see that the resolution of the domain wall problem requires a small value for the Higgs-singlet coupling besides the large enough Hubbleinduced mass.

\section{COSMOLOGICAL CONSEQUENCES FOR ELECTROWEAK BARYOGENESIS AND DARK MATTER}

The results obtained in this paper lead to several consequences for cosmology in the NMSSM. First, for sufficiently small couplings the singlet sector would decouple from the thermal bath. A dominant interaction process between the singlet fields and the MSSM fields is the $2 \rightarrow 2$ interaction involving Higgsinos and weak bosons (and their SUSY partners), whose rate is roughly estimated as $\Gamma \sim \alpha \lambda^{2} T$, where $\alpha$ is the weak coupling strength. Since this interaction rate is exponentially suppressed after the temperature of the thermal bath becomes less than $m_{S} \sim \mathcal{O}(\mathrm{TeV})$, and the Hubble parameter $H \propto T^{2}$ decays faster than $\Gamma$, we expect that the fields in the singlet sector would never thermalize if the condition $\Gamma<H$ is satisfied at $T \sim m_{S}$. This occurs if the value of the coupling satisfies

$$
\lambda<\alpha^{-\frac{1}{2}}\left(\frac{m_{S}}{M_{\mathrm{Pl}}}\right)^{\frac{1}{2}} \sim \mathcal{O}\left(10^{-7}-10^{-6}\right) .
$$

For such a small coupling the singlino state cannot be produced from the thermal bath, and it would not contribute to the dark matter abundance. Finally, the $S$ field falls in the global minimum much earlier than the epoch of the electroweak phase transition $T \sim \mathcal{O}(100) \mathrm{GeV}$, if the tracking mechanism works. Therefore, the first order phase transition is not likely to be realized in this case. These facts would have an important consequence for the electroweak baryogenesis within NMSSM and the mass range for the dark matter, which would now primarily contain the Higgsino component [40].

\section{CONCLUSION AND DISCUSSIONS}

In this paper, we have shown that the domain wall problem of the NMSSM can be resolved via the tracking mechanism if the following conditions are satisfied: (1) There exist negative Hubble-induced corrections with sufficiently large coefficients [see Eq. (18)] in the effective potential for the $S$ field during and after inflation. (2) The $A_{\kappa}$ term is slightly larger than the soft mass $m_{S}$ [see Eq. (3)] in order to prevent the $S$ field from rotating in the phase direction at later times. (3) The thermal effects must remain irrelevant; see Eq. (30). This requirement leads to the constraints on the coupling strength and the reheating temperature as shown in Fig. 2. Our study has many important phenomenological consequences for LHC and dark matter creation and detection within NMSSM.

Let us briefly mention a particular relevance for the future experimental studies of NMSSM. Typically, in NMSSM the Higgs sector is enlarged due to the presence of a singlet, and for reasonably large couplings, i.e., $\lambda, \kappa \sim 0.1$, Higgs-to-Higgs decays can possibly be observed in the forthcoming LHC experiment [41]. According to Fig. 2, such a discovery at a large coupling regime $\lambda \sim$ $\mathcal{O}(0.1)$ indicates the formation of domain walls; then we must seriously take into account their cosmological evolution $[42,43]$.

Another important LHC signature would be obtained in the small coupling regime, where the singlet states decouple from the MSSM sector. In this case, we would expect to 
observe displaced vertices from the long-lived next-to-LSP [44], which can also be helpful to falsify our scenario.

\section{ACKNOWLEDGMENTS}

A. M. thanks Csaba Balasz for a discussion about some aspects of NMSSM. K. S. thanks Koichi Hamaguchi, Kyohei Mukaida, Kazunori Nakayama, and Osamu Seto for a discussion about the stability of the tracking behavior of the singlet field. A. M. and M. Y. thank the Japan Society for the Promotion of Science (JSPS) Invitation Fellowship for Research in Japan. A. M. is supported by the STFC Grant No. ST/J000418/1. K. S. is supported by the JSPS through research fellowships. This work was in part supported by the JSPS Grant-in-Aid for Scientific Research Nos. 25287054 (M. Y.), 26610062 (M. Y.), $15 \mathrm{H} 02082$ (J. Y.), and the JSPS Grant-in-Aid for Scientific Research on Innovative Areas No. 15H05888 (M. Y. and J. Y.).
[1] H. P. Nilles, Phys. Rep. 110, 1 (1984).

[2] S. P. Martin, Adv. Ser. Dir. High Energy Phys. 21, 1 (2010).

[3] K. Enqvist and A. Mazumdar, Phys. Rep. 380, 99 (2003).

[4] G. Jungman, M. Kamionkowski, and K. Griest, Phys. Rep. 267, 195 (1996).

[5] D. H. Lyth and A. Riotto, Phys. Rep. 314, 1 (1999).

[6] A. Mazumdar and J. Rocher, Phys. Rep. 497, 85 (2011).

[7] M. Yamaguchi, Classical Quantum Gravity 28, 103001 (2011).

[8] H. Murayama, H. Suzuki, T. Yanagida, and J. Yokoyama, Phys. Rev. Lett. 70, 1912 (1993).

[9] H. Murayama, H. Suzuki, T. Yanagida, and J. Yokoyama, Phys. Rev. D 50, R2356 (1994).

[10] K. Kadota and J. Yokoyama, Phys. Rev. D 73, 043507 (2006).

[11] R. Allahverdi, K. Enqvist, J. Garcia-Bellido, and A. Mazumdar, Phys. Rev. Lett. 97, 191304 (2006).

[12] R. Allahverdi, K. Enqvist, J. Garcia-Bellido, A. Jokinen, and A. Mazumdar, J. Cosmol. Astropart. Phys. 06 (2007) 019.

[13] K. Kamada and J. Yokoyama, Prog. Theor. Phys. 122, 969 (2009).

[14] S. Ferrara, R. Kallosh, A. Linde, A. Marrani, and A. Van Proeyen, Phys. Rev. D 82, 045003 (2010).

[15] S. Ferrara, R. Kallosh, A. Linde, A. Marrani, and A. Van Proeyen, Phys. Rev. D 83, 025008 (2011).

[16] I. Affleck and M. Dine, Nucl. Phys. B249, 361 (1985).

[17] H. P. Nilles, M. Srednicki, and D. Wyler, Phys. Lett. B 120B, 346 (1983); J. Frère, D. R. T. Jones, and S. Raby, Nucl. Phys. B222, 11 (1983); J. P. Derendinger and C. A. Savoy, Nucl. Phys. B237, 307 (1984).

[18] J. E. Kim and H. P. Nilles, Phys. Lett. B 138B, 150 (1984).

[19] U. Ellwanger, C. Hugonie, and A. M. Teixeira, Phys. Rep. 496, 1 (2010).

[20] J. A. Frieman, C. T. Hill, and R. Watkins, Phys. Rev. D 46, 1226 (1992).

[21] R. Brandenberger, D. A. Easson, and A. Mazumdar, Phys. Rev. D 69, 083502 (2004).
[22] Y. Zeldovich, I. Y. Kobzarev, and L. Okun, Zh. Eksp. Teor. Fiz. 67, 3 (1974).[Sov. Phys. JETP 40, 1 (1975)].

[23] C. Panagiotakopoulos and K. Tamvakis, Phys. Lett. B 446, 224 (1999).

[24] J. McDonald, Nucl. Phys. B530, 325 (1998).

[25] U. Ellwanger, M. Rausch de Traubenberg, and C. A. Savoy, Nucl. Phys. B492, 21 (1997).

[26] A. Vilenkin and L. H. Ford, Phys. Rev. D 26, 1231 (1982).

[27] A. D. Linde, Phys. Lett. B 116B, 335 (1982).

[28] A. A. Starobinsky, Phys. Lett. B 117B, 175 (1982).

[29] A. A. Starobinsky and J. Yokoyama, Phys. Rev. D 50, 6357 (1994).

[30] L. Kofman, A. D. Linde, and A. A. Starobinsky, Phys. Rev. Lett. 73, 3195 (1994).

[31] K. Sato and J. Yokoyama, Int. J. Mod. Phys. D g24, 1530025 (2015).

[32] M. Dine, L. Randall, and S. D. Thomas, Phys. Rev. Lett. 75, 398 (1995); M. Dine, L. Randall, and S. D. Thomas, Nucl. Phys. B458, 291 (1996).

[33] S. Choudhury, A. Mazumdar, and E. Pukartas, J. High Energy Phys. 04 (2014) 077.

[34] A. D. Linde, Phys. Rev. D 53, R4129 (1996).

[35] J. M. Bardeen, J. R. Bond, N. Kaiser, and A. S. Szalay, Astrophys. J. 304, 15 (1986).

[36] J. Yokoyama, Phys. Rev. D 58, 107502 (1998).

[37] E. W. Kolb and M. S. Turner, Front. Phys. 69, 1 (1990).

[38] M. Kawasaki and T. Takesako, Phys. Lett. B 711, 173 (2012).

[39] D. H. Lyth and T. Moroi, J. High Energy Phys. 05 (2004) 004.

[40] C. Balazs, A. Mazumdar, E. Pukartas, and G. White, J. High Energy Phys. 01 (2014) 073.

[41] N. D. Christensen, T. Han, Z. Liu, and S. Su, J. High Energy Phys. 08 (2013) 019.

[42] S. Abel, S. Sarkar, and P. White, Nucl. Phys. B454, 663 (1995)

[43] K. Kadota, M. Kawasaki, and K. Saikawa, J. Cosmol. Astropart. Phys. 10 (2015) 041.

[44] U. Ellwanger and C. Hugonie, Eur. Phys. J. C 5, 723 (1998). 